\title{
Probing neutrino mass with displaced vertices at the Tevatron
}

\author{
F. de Campos, ${ }^{1, *}$ O. J. P. Éboli, ${ }^{2, \dagger}$ M. B. Magro, ${ }^{2,}+\ddagger$ \\ W. Porod, ${ }^{3,4, \S}$ D. Restrepo, $, 3,5$ and J. W. F. Valle ${ }^{3, * *}$ \\ ${ }^{1}$ Departamento de Física e Química, Universidade Estadual Paulista, Guaratinguetá - SP, Brazil \\ ${ }^{2}$ Instituto de Física, Universidade de São Paulo, São Paulo - SP, Brazil. \\ ${ }^{3}$ Instituto de Física Corpuscular - C.S.I.C./Universitat de València \\ Edificio Institutos de Paterna, Apt 22085, E-46071 València, Spain \\ ${ }^{4}$ Inst. für Theoretische Physik, Uni. Zürich \\ ${ }^{5}$ Instituto de Física, Universidad de Antioquia - Colombia
}

\begin{abstract}
Supersymmetric extensions of the standard model exhibiting bilinear R-parity violation can generate naturally the observed neutrino mass spectrum as well mixings. One interesting feature of these scenarios is that the lightest supersymmetric particle (LSP) is unstable, with several of its decay properties predicted in terms of neutrino mixing angles. A smoking gun of this model in colliders is the presence of displaced vertices due to LSP decays in large parts of the parameter space. In this work we focus on the simplest model of this type that comes from minimal supergravity with universal R-parity conserving soft breaking of supersymmetry (RmSUGRA). We evaluate the potentiality of the Fermilab Tevatron to probe the RmSUGRA parameters through the analysis of events possessing two displaced vertices stemming from LSP decays. We show that requiring two displaced vertices in the events leads to a reach in $m_{1 / 2}$ twice the one in the usual multilepton signals in a large fraction of the parameter space.
\end{abstract}

\footnotetext{
*Electronic address: fernando@ift.unesp.br

${ }^{\dagger}$ Electronic address: eboli@fma.if.usp.br

${ }^{\ddagger}$ Electronic address: magro@fma.if.usp.br

§Electronic address: porod@ific.uv.es

๑Electronic address: restrepo@uv.es

**Electronic address: valle@ific.uv.es
} 


\section{INTRODUCTION}

Despite the lack of direct experimental evidence, supersymmetry is the most popular candidate for physics beyond the standard model (SM). Apart from its known motivations related in particular to the hierarchy problem, supersymmetry may provide also the understanding of the origin of neutrino mass [1]. Supersymmetric (SUSY) models have been used as benchmarks to evaluate the potentiality for new discoveries at the current high energy colliders, such as the Tevatron, and the future colliders, like the CERN Large Hadron Collider or the International Linear Collider. Collider signals for supersymmetry have been studied both in the context of R-parity conservation [2, 3, 4, 5], as well as in scenarios with $\mathrm{R}$-parity violation [6, 7, 8, ㅇ, 10].

A variety of recent neutrino physics experiments [11] has established the existence of neutrino oscillations and masses, indicating clearly the need for physics beyond the SM. It is tempting to imagine that neutrino physics and supersymmetry are tied together [12]: SUSY models exhibiting R-parity violation can lead to a pattern of neutrino masses and mixings [13] in agreement with the current solar and reactor neutrino data as well as atmospheric and accelerator neutrino data. The simplest realization of this idea assumes bilinear violation of $\mathrm{R}$-parity [14, 15]. Such restricted $\mathrm{R}$-parity violation can arise either as an effective description of a spontaneous $\mathrm{R}$-parity violation scenario [16], or as a result of suitable symmetries of the underlying theory [17]. It also corresponds to the simplest broken $\mathrm{R}-$ parity version of the Minimal Supersymmetric Standard Model (MSSM), to which we refer, in what follows, as RMSSM.

Despite the presence of additional interactions in $\mathrm{R}$-parity violating supersymmetry, the SUSY particle production cross sections at colliders are essentially the same as in the MSSM, since the smallness of neutrino masses implies small $\mathrm{R}$-parity violation parameters. Similarly, the SUSY particle cascade decays are controlled by R-parity conserving interactions until the LSP is produced. In contrast to R-parity conserving supersymmetry, the LSP is unstable in these models as it is not protected by any symmetry. In fact, R-parity violating interactions determine its branching ratios and can lead to large decay lengths and displaced vertices if these interactions are sufficiently small. Parenthetically, let us mention that a light gravitino, as it occurs for example gauge mediated SUSY breaking, is a possible

candidate for dark matter [18]. In case that the gravitino is heavy, dark matter must come 
from some other source, like the QCD-sector axion. In the light gravitino case displaced vertices discussed here should come from the R-parity violating NLSP decays which still have a large probability [19]. Turning to the case of interest, namely the RMSSM, it has been shown that in this case the LSP decay properties can be directly tested at collider experiments [7, 8, 19, 20, 21, 22, 23].

In this work, we focus on the possibility of the $\tilde{\chi}_{1}^{0}$ decay length being large enough to generate displaced vertices at the Tevatron, which could be observed by the tracking systems of the experimental collaborations. We work within the framework of the simplest model of this kind, namely the minimal supergravity (mSUGRA) version of the RMSSM with universal soft breaking terms at unification [13]. We call it RmSUGRA, for short. However, here we are not enforcing universality conditions for R-parity violating soft terms. In such models one can show that over a large range of the accessible parameter space the masses of the sleptons, the lighter chargino $\left(\tilde{\chi}_{1}^{ \pm}\right)$, and the lighter neutralinos $\left(\tilde{\chi}_{1}^{0}\right.$ and $\left.\tilde{\chi}_{2}^{0}\right)$ are considerably smaller than the gluino and squark masses; see e.g. 24] and references therein. As a result, at Tevatron energies, the largest reach in supersymmetry searches comes from the production of charginos and neutralinos, and one might expect a copious number of displaced vertex events at the Tevatron depending on the parameters of the model [7].

In this paper we show that the presence of bilinear $\mathrm{R}$-parity violating $(\mathrm{BRpV})$ interactions enhances the discovery reach for supersymmetry over most of the parameter space with respect to the one obtained using the trilepton/multilepton signal [8, 25]. Previous analysis of the potential of the Tevatron for probing neutrino mass models based on $\mathrm{R}$-parity violation either analyzed the changes in the multilepton signal due to the LSP decay [8] or studied the same sign dilepton production presenting a displaced vertex due to the decay of a neutralino into $\mu W$ or $\tau W$ pairs [7]. Moreover, these analyses were performed imposing only the constraints from atmospheric neutrino data. Here, we analyzed events exhibiting two reconstructed displaced vertices and we scan the full accessible RmSUGRA parameter space at Tevatron, taking into account all constraints coming from neutrino masses and mixing angles in the $\mathrm{BRpV}$ model. We show that the predicted number of events at Tevatron depends mainly on $m_{1 / 2}$, or equivalently the neutralino mass, with mild dependences on the other mSUGRA parameters or specific neutralino decay channels.

This paper is organized as follows: Section [1] summarizes the most relevant features of the RmSUGRA model. The displaced vertex analysis is presented in section III, while we 
summarize our conclusions in section IV.

\section{MAIN FEATURES OF THE RMSUGRA MODEL}

Here we consider a simple variant of the MSSM that includes the following bilinear terms in the superpotential [14, 15, 26]

$$
W_{\mathrm{BRpV}}=W_{\mathrm{MSSM}}+\varepsilon_{a b} \epsilon_{i} \widehat{L}_{i}^{a} \widehat{H}_{u}^{b}
$$

The relevant bilinear terms in the soft supersymmetry breaking sector are

$$
V_{\mathrm{soft}}=m_{H_{u}}^{2} H_{u}^{a *} H_{u}^{a}+m_{H_{d}}^{2} H_{d}^{a *} H_{d}^{a}+M_{L_{i}}^{2} \widetilde{L}_{i}^{a *} \widetilde{L}_{i}^{a}-\varepsilon_{a b}\left(B \mu H_{d}^{a} H_{u}^{b}+B_{i} \epsilon_{i} \widetilde{L}_{i}^{a} H_{u}^{b}\right)
$$

The simultaneous presence of the bilinear term in the superpotential and its analogue in the soft supersymmetry breaking sector violates $\mathrm{R}$-parity and lepton number explicitly, inducing vacuum expectation values (vev) $v_{i}, i=1,2,3$ for the sneutrinos. In order to reproduce the

observed values of neutrino masses and mixings [11] we must have $\left|\epsilon_{i}\right| \ll|\mu|$, where $\mu$ denotes the SUSY bilinear mass parameter [13].

In a minimal supergravity model with universal soft breaking terms at unification the relevant parameters are

$$
m_{0}, m_{1 / 2}, \tan \beta, \operatorname{sign}(\mu), A_{0}, \epsilon_{i}, \text { and } \Lambda_{i}
$$

where $m_{1 / 2}$ and $m_{0}$ are the common gaugino mass and scalar soft SUSY breaking masses at the unification scale, $A_{0}$ is the common trilinear term, and $\tan \beta$ is the ratio between the Higgs field vev's. Although many parameterizations are possible, we take as the free parameters the $\epsilon_{i}$ of the superpotential and the so-called alignment parameters, $\Lambda_{i}=\epsilon_{i} v_{d}+$ $\mu v_{i}$. Moreover, we are not enforcing universality conditions for $\mathrm{R}$-parity violating soft terms.

Successful explanation of neutrino data requires small $\mathrm{R}$-parity violating couplings. Therefore, the induced shift in the SUSY masses by the BRpV interactions is smaller than the foreseeable experimental accuracy, and thus, we use the following strategy: for a given set of the $\mathrm{R}$-parity conserving parameters we calculate the mass spectrum setting the $\mathrm{R}-$ parity violating parameters to zero when evolving the parameters from the unification scale to the electroweak scale. This procedure was carried out using the package SPheno; details of this calculation can be found in [27]. At the electroweak scale we choose the R-parity 
violating parameters such that the neutrino data are successfully explained within the $3 \sigma$ range as given,e.g. in Ref. [11]:

$$
\begin{aligned}
& 0.23 \lesssim \sin ^{2} \theta_{\text {sol }} \lesssim 0.38, \\
& 7.1 \times 10^{-5} \mathrm{eV}^{2} \lesssim \Delta m_{\text {sol }}^{2} \lesssim 8.9 \times 10^{-5} \mathrm{eV}^{2} \text {, } \\
& 0.34 \lesssim \sin ^{2} \theta_{\text {atm }} \lesssim 0.68, \\
& 1.4 \times 10^{-3} \mathrm{eV}^{2} \lesssim \Delta m_{\mathrm{atm}}^{2} \lesssim 3.3 \times 10^{-3} \mathrm{eV}^{2} .
\end{aligned}
$$

In order to obtain the mixing matrices between SM and SUSY particles we diagonalize the corresponding mass matrices containing SM and SUSY fermions and scalars, for instance, the full $7 \times 7$ mass matrix for neutrinos and neutralinos. Afterwards all SUSY R-parity violating as well as $\mathrm{R}$-parity conserving decays are calculated using an adapted version of SPheno. The output of this calculation is tabulated in the SLHA format [28] and inputed into PYTHIA [29], which is used to generate the events.

We studied the scenarios where the LSP is the lightest neutralino which decays into SM particles due to the mixings induced by the $\mathrm{BRpV}$ interactions. The lightest neutralino has leptonic decays $\tilde{\chi}_{1}^{0} \rightarrow \nu \ell^{+} \ell^{\prime-}$, the invisible mode $\tilde{\chi}_{1}^{0} \rightarrow \nu \nu \nu$ and semi-leptonic decays $\tilde{\chi}_{1}^{0} \rightarrow \nu q \bar{q}, \ell q \bar{q}$ [21]. As an illustration, we depict in Fig. 团 the lightest neutralino branching ratios as a function of $m_{0}$ for $m_{1 / 2}=350 \mathrm{GeV}, A_{0}=-100 \mathrm{GeV}, \mu>0$, and $\tan \beta=10$. In this plot we took the $\mathrm{R}$-parity violating parameters for each mSUGRA point that lead to the largest decay length compatible with neutrino data at the $3 \sigma$ level. As we can see from this figure, the decay $\tilde{\chi}_{1}^{0} \rightarrow \nu b \bar{b}$ dominates at small $m_{0}$. This decay channel arises from an effective coupling $\lambda_{i 33}^{\prime} \sim\left(\epsilon_{i} / \mu\right) h_{b}$, induced by the Higgs-slepton mixing, where $h_{b}$ is the bottom Yukawa coupling. Therefore, this decay is enhanced at small $m_{0}$ due to the lightness of the scalars mediating it and at large $\tan \beta$ due to enhanced Yukawa couplings. Moreover, at large $m_{0}$ all $\tilde{\chi}_{1}^{0}$ decay channels give a sizeable contribution with the main decay modes being the semi-leptonic ones exhibiting light quarks.

The expected $\tilde{\chi}_{1}^{0}$ lifetime (decay length) depends both on the magnitude of $\mathrm{R}$-parity breaking parameters and the chosen values of the mSUGRA parameters. The smallness of the $\mathrm{R}$-parity violating parameters implies that the lightest neutralino has a small decay width 21], thus having a lifetime large enough to give rise to displaced vertices. In Fig. 22 we depict the lightest neutralino decay length as a function of $m_{1 / 2}$ for $m_{0}=100,200$, and 1000 $\mathrm{GeV}$ and $A_{0}=-100, \mu>0, \tan \beta=10$. In this figure, we varied the $\mathrm{R}-$ parity violating 
parameters so that $|\vec{\epsilon} / \mu|$ and $|\vec{\Lambda}|^{2}$ lie in the $3 \sigma$ bands required to account for the neutrino oscillation data [11]. Note from Fig. 2 that the LSP decay length strongly depends on $m_{0}$, as it controls the masses of the scalar particles exchanged in the LSP decay. In contrast, the dependence on $A_{0}$ is rather mild. One can see from this figure that the LSP decay length is in the range of tenths of $\mathrm{mm}$ up to $\simeq 10 \mathrm{~cm}$. Therefore, the lightest neutralino should decay inside the detectors of Tevatron yielding an observable displaced vertex.

\section{SIGNAL, BACKGROUNDS, AND SELECTION CUTS}

In the context of our RMSSM/RmSUGRA, supersymmetric particle production and subsequent cascade decays proceed as in the R-parity conserving case (MSSM/mSUGRA) up to the final state containing two lightest neutralinos, as mentioned above. Since neutrino data require the $\mathrm{R}$-parity violating couplings to be small, the decays of these two neutralinos leads, in general, to displaced vertices [21]. Assuming that gluinos and squarks are too heavy to be produced at the Tevatron, the most important SUSY production processes at the Tevatron are

$$
p \bar{p} \rightarrow \tilde{\ell}^{+} \tilde{\ell}^{-} \quad, \quad \tilde{\nu} \tilde{\ell} \quad, \quad \tilde{\chi}_{i}^{0} \tilde{\chi}_{j}^{0}(i(j)=1,2) \quad, \quad \tilde{\chi}_{1}^{+} \tilde{\chi}_{1}^{-} \quad \text { and } \quad \tilde{\chi}_{i}^{0} \tilde{\chi}_{1}^{ \pm}(i=1,2)
$$

The main decay modes of the lightest neutralino in our model are

- $\tilde{\chi}_{1}^{0} \rightarrow \nu \ell^{+} \ell^{-}$with $\ell=e, \mu$ denoted by $\ell \ell$;

- $\tilde{\chi}_{1}^{0} \rightarrow \nu q \bar{q}$ denoted $j j$;

- $\tilde{\chi}_{1}^{0} \rightarrow \tau q^{\prime} \bar{q}$, called $\tau j j$;

- $\tilde{\chi}_{1}^{0} \rightarrow \nu b \bar{b}$, that we denote by $b b$;

- $\tilde{\chi}_{1}^{0} \rightarrow \nu \tau^{+} \tau^{-}$, called $\tau \tau$;

- $\tilde{\chi}_{1}^{0} \rightarrow \tau \nu \ell$, called $\tau \ell$.

In order to mimic the triggers used by the Tevatron collaborations, we accept events passing at least one of the following requirements:

1. the event has two muons with transverse momenta satisfying $p_{T}>4 \mathrm{GeV}$; 
2. the event possesses a lepton (electron or muon) with $p_{T}>4 \mathrm{GeV}$ and a displaced track with an impact parameter in excess of $0.120 \mathrm{~mm}$ and $p_{T}>2 \mathrm{GeV}$;

3. the event exhibits two displaced tracks of opposite charge with $p_{T}>3 \mathrm{GeV}$ and impact parameter larger than $0.120 \mathrm{~mm}$.

In the $\ell \ell, j j, \tau j j$, and $b b$ decays, the tracks point to the LSP decay vertex, up to measurement errors. In our analysis we smeared the energies, but not directions, of all final state particles with a Gaussian error given by $\Delta E / E=0.7 / \sqrt{E}(E$ in $\mathrm{GeV})$ for hadrons and $\Delta E / E=0.15 / \sqrt{E}$ for charged leptons. Therefore, the LSP decay vertex is reconstructed correctly for these modes within these approximations. On the other hand, the tracks originating from the $\tau \tau$ and $\tau \ell$ decay modes do not converge to the LSP decay vertex. For these events we looked for tracks that do not point to the interaction region and whose reconstructed trajectories come closer than $100 \mu \mathrm{m}(1000 \mu \mathrm{m})$ if the closest point of the two trajectories are inside the silicon detector (central tracker). In this case, we defined the position of the displaced vertex as the average of the closest points of the two trajectories.

In our analysis, we also required that the two reconstructed vertices are away from the interaction point, more specifically, we demanded that both LSP decay vertices lie outside an ellipsoid

$$
\left(\frac{x}{\delta_{x y}}\right)^{2}+\left(\frac{y}{\delta_{x y}}\right)^{2}+\left(\frac{z}{\delta_{z}}\right)^{2}=1
$$

where the $z$-axis is along the beam direction. We assumed that $\delta_{x y}=150 \mu \mathrm{m}$ and $\delta_{z}=$ $300 \mu \mathrm{m}$.

To guarantee a high efficiency in the reconstruction of the displaced vertices without a full detector simulation, we restricted our analysis to reconstructed vertices with pseudorapidities $|\eta|<1.5$. Moreover, both neutralinos should decay well inside the inner detectors to guarantee enough hits in the track system. Therefore, we require that the neutralinos decay inside a cylinder with radius $r=350 \mathrm{~mm}$ and length $l=1250 \mathrm{~mm}$.

The SM backgrounds coming, for instance, from displaced vertices associated to $b$ 's or $\tau$ 's can be eliminated by requiring that the set of tracks defining a displaced vertex should have an invariant mass larger than $20 \mathrm{GeV}$. This way the displaced vertex signal passing all the above cuts is essentially background free. Fig. [ displays the reconstructed invariant mass of displaced vertices after cuts for $m_{0}=400 \mathrm{GeV}, m_{1 / 2}=350 \mathrm{GeV}, A_{0}=-100 \mathrm{GeV}, \mu>0$, $\tan \beta=10$ and $|\boldsymbol{\epsilon}| / \mu$ taking the largest value compatible with neutrino data. As we can 
see, the neutralino decays lead to a substantial invariant mass associated to the displaced vertices. If necessary, the invariant mass can be further tightened without a substantial loss of signal.

We display in Fig. 4 the expected number of events $(N E v)$ after the above cuts as function of the neutralino decay length for various values of $m_{0}$ and $m_{1 / 2}$ and an integrated luminosity of $8 \mathrm{fb}^{-1}$. In this plot we chose the $\mathrm{R}$-parity violating parameters such that the largest decay length is obtained compatible with neutrino data at the $3 \sigma$ level. The same plot for smaller decay lengths is basically the same but with a shift of large $m_{0}$ values to the left side. For example, for the point with $\left(m_{1 / 2}, m_{0}\right)=(350,1000) \mathrm{GeV}$ we have $N E v=21$, $L\left(\tilde{\chi}_{1}^{0}\right)=0.9 \mathrm{~mm}$ and $|\boldsymbol{\epsilon} / \mu|=5.2 \times 10^{-4}$, while for smaller value of $|\boldsymbol{\epsilon} / \mu|=4 \times 10^{-4}$ for the same SUGRA point, we find $N E v=18.4$ and $L\left(\tilde{\chi}_{1}^{0}\right)=0.74 \mathrm{~mm}$. In general, the variation of the number of events at any SUGRA point is of order $10 \%$ for the full range for the R-parity parameters compatible with neutrino data. The reason for this behaviour is that the decay length is mainly determined by the values of the neutrino masses which are already known rather precisely.

In order to illustrate the behavior of the signal for a fixed value of $m_{0}$ we present, in Fig. 5, the expected number of events for the same parameters as in Fig. 4 but for fixed $m_{0}=100 \mathrm{GeV}$. The numbers given beside some points indicate the corresponding value of $m_{1 / 2}$. At small decay lengths $(\lesssim 0.3 \mathrm{~mm})$ the production cross section of neutralinos is rather low, as well as the efficiency for extracting the signal. As the decay length increases, $m_{1 / 2}$ $\left(m_{\tilde{\chi}_{1}^{0}}\right)$ decreases and the production cross section increases, however, the detection efficiency reaches a maximum around $\simeq 1 \mathrm{~mm}$ and then decreases again. These two opposite behaviors lead to an approximately constant signal rate for decay lengths $\gtrsim 10 \mathrm{~mm}$.

We are now set to study the discovery reach of the Tevatron using the displaced vertex signal. In Fig. [6 we show the region of the $m_{0} \otimes m_{1 / 2}$ plane where the displaced vertex signal can be established at Tevatron for integrated luminosities $\mathcal{L}$ of $2 \mathrm{fb}^{-1}$ and $8 \mathrm{fb}^{-1}$ and fixed values of $A_{0}, \tan \beta$, and $\operatorname{sign}(\mu)(>0)$. Our conventions are as follows: in the points below the solid line the expected number of events $(N E v)$ is greater than 4 , while the region below the dashed line exhibits a sizeable statistics $(N E v>15)$. The black squares denote points with an expected number of events greater than 4 for an integrated luminosity of $8 \mathrm{fb}^{-1}$, while the grey (green) squares indicates the points presenting a high statistics $(N E v>15)$ for this luminosity. Moreover, points denoted by diamonds have $2<N E v<4$ with $8 \mathrm{fb}^{-1}$, 
while the stars correspond to points with $N E v<2$ for $\mathcal{L}=8 \mathrm{fb}^{-1}$. The present direct search limits are violated at the points indicated with a round black circle, while the white circles mark the points where the neutralino is no longer the LSP but the lighter stau. The stau is to short-lived to produce a visible decay length as has been shown in 22]. Therefore a different analysis is required for such cases.

In Fig. [6(a), top-left panel, we present the Tevatron reach for $A_{0}=-100 \mathrm{GeV}, \tan \beta=10$ and $\mu>0$, taking the the $\mathrm{R}$-parity violating parameters such that we obtain the largest decay length for the lightest neutralino and being at the same time compatible at $3 \sigma$ level with the present neutrino data. We see from this figure that the Tevatron with $2 \mathrm{fb}^{-1}$ integrated luminosity is able to probe $m_{1 / 2} \lesssim 350 \mathrm{GeV}$ at moderate and large values of $m_{0}$. At small values of $m_{0}$, where the decay lengths are smaller and the main decay channel is $b \bar{b} \nu$, the Tevatron can probe well the region $m_{1 / 2} \lesssim 310 \mathrm{GeV}$. This reach is larger than the one provided by trilepton and multilepton searches [8]. Moreover, for $m_{1 / 2} \lesssim 300 \mathrm{GeV}$ and $m_{0} \gtrsim 300 \mathrm{GeV}$ we expect a large number of events, which can not only make the discovery easy, but also be used to perform preliminary studies of the neutralino branching ratios and confront them with the predictions of our model. The Tevatron reach can be considerably extended provided larger integrated luminosities become available, for instance, the region $m_{1 / 2} \lesssim 410 \mathrm{GeV}$ and large values of $m_{0}$ can be explored for $\mathcal{L}=8 \mathrm{fb}^{-1}$ with the region $m_{1 / 2} \lesssim 350 \mathrm{GeV}$ exhibiting a large statistics.

In order to understand the effect of different choices of the $\mathrm{R}$-parity violating parameters, we present in Fig. 6(b), top-right panel, the Tevatron reach for R-parity violating parameters leading to a minimal decay length of the lightest neutralino compatible with neutrino data while keeping the R-parity conserving parameters fixed. As expected from the previous discussion, the reach is in both cases rather similar.

To study how our results depend upon $A_{0}$, we present in Fig. 6(c), lower-left panel, the displaced vertex discovery reach at the Tevatron for $A_{0}=-900 \mathrm{GeV}$ and all other parameters as in Fig. [6(a) and the R-parity violating parameters such that the neutralino decay length is maximized. The changes in the predicted number of events are again at the level of a few percent which leads to slightly smaller Tevatron reach. Furthermore, there is a region with a stau LSP which cannot be covered by the present analysis. In Fig. [6(d) the mSUGRA parameters are chosen as in (a) but with $\tan \beta=40$. Again we have chosen the $\mathrm{R}$-parity violating parameters such that the neutralino decay length is maximized. As we 
can see, the Tevatron reach increases at moderate $m_{0}$, remaining approximately the same at large $m_{0}$. As in the previous case, Fig. 6(c), the stau is the LSP at small $m_{0}$ and thus this region is not covered by our analysis.

We can learn from the Fig. [6 panels that the displaced vertex signal remains approximately stable, showing that this channel depends mainly upon $m_{1 / 2}$ and hardly on the other parameters, including the $\mathrm{R}$-parity violating ones provided they are in the range allowed by neutrino data. This is at variance with the behavior of the trilepton and multilepton signals for our R-parity violating SUGRA model [8, 9]. The reason for this difference is that the decay length depends, in addition to $m_{\tilde{\chi}_{1}^{0}}$, mainly upon the two ratios $|\boldsymbol{\epsilon} / \mu|$ and $|\boldsymbol{\Lambda}|^{2} / \operatorname{det}\left(m_{\tilde{\chi}^{0}}\right) \mid$, which are constrained to lie in narrow ranges by the neutrino data [13]. In contrast, as seen in Fig. 11, the neutralino branching ratios into different allowed channels exhibits a much stronger dependence on the RmSUGRA parameters [8, 9] being, therefore, less robust.

For illustrative purpose only, as the maximum luminosity expected for the Tevatron RUNII will be at most $8 \mathrm{fb}^{-1}$, we present in Fig. 7 the expected reach in the displaced vertex channel assuming an integrated luminosity of $25 \mathrm{fb}^{-1}$ in order to allow easier comparison with the Tevatron reach of the trilepton signal in the MSSM 24] and the trilepton and multilepton signals with RmSUGRA obtained in Ref. [8]. Comparing the last two signals we learn that the presence of $\mathrm{R}$-parity violation enhances the Tevatron reach over most of the parameter space, specially at moderate and large $m_{0}$. Moreover, the reach in $m_{1 / 2}$ of the displaced vertex signal is more than twice the one of the trilepton and multilepton signals for basically all values of $m_{0}$ and $\tan \beta$.

Despite the large reach of the two-displaced-vertices channel, it is interesting to study whether we can further enlarge the Tevatron sensitivity to RmSUGRA. One possible way to achieve this goal is to consider events containing one or more displaced vertices 7] that pass our selection cuts instead of requiring two displaced vertices. We depicted in Fig. 8 the Tevatron reach requiring at least one reconstructed vertex in the events for the same choice of parameters as in Fig. 6(a). As we can see, the reach in $m_{1 / 2}$ can be extended in $50 \mathrm{GeV}$ in accordance with ref. [7]. However, this results should be taken with a pinch of salt since it is not obvious that our cuts eliminate completely the SM backgrounds when there is just one observed displaced vertex. To verify this result a detailed detector simulation is called for. 


\section{CONCLUSION}

We have analyzed displaced vertices from neutralino decays in a supergravity model with violation of R-parity (RmSUGRA) at the Fermilab Tevatron. In this model R-parity is violated effectively by bilinear terms in the superpotential and soft supersymmetry breaking sector. Despite the small $\mathrm{R}$-parity violating couplings needed to generate the neutrino masses and mixings indicated by current neutrino data, the lightest supersymmetric particle can decay inside the detector. This leads to a phenomenology quite distinct from that of the $\mathrm{R}$-parity conserving scenario. We have quantified the Tevatron reach for the displaced vertices signal, displaying our results in the $m_{0} \otimes m_{1 / 2}$ plane.

In contrast with other studies [7], we have a background free signal with a very stable yield which depends mainly on the neutralino mass. This follows from our requirement of

two displaced vertices instead of only one 7], and the fact that we imposed all constraints of the neutrino data on the RmSUGRA parameters. In fact, even for $m_{1 / 2} \approx 470 \mathrm{GeV}$ $\left(m_{\tilde{\chi}_{1}^{0}} \approx 215 \mathrm{GeV}\right)$, and moderate to high $m_{0}$ values, a few events are expected for an integrated luminosity of $8 \mathrm{fb}^{-1}$, with a minor dependence in other RmSUGRA parameters. This reach is considerably larger than the one in the trilepton/multilepton channels in either the MSSM or RmSUGRA. Therefore, the displaced-vertices signal is an essential tool to extend considerable the search possibilities of Tevatron in the case of broken R-parity.

\section{Acknowledgments}

We thank A. Garcia-Bellido for discussions. This work was supported by Spanish grant BFM2002-00345, by the European Commission Human Potential Program RTN network MRTN-CT-2004-503369, by Conselho Nacional de Desenvolvimento Científico e Tecnológico $(\mathrm{CNPq})$, and by Fundação de Amparo à Pesquisa do Estado de São Paulo (FAPESP). W. P. has been supported by a MCyT Ramon y Cajal contract and partly by the 'Swiss Nationalfonds'. D.R is supported by "Secretaría de Estado de Educación y Universidades" 
postdoc contract SB2001-0056

[1] For a recent review see, M. Hirsch and J. W. F. Valle, "Supersymmetric origin of neutrino mass," New J. Phys. 6, 76 (2004) hep-ph/0405015.

[2] J. Amundson et al., Proceedings of the 1996 DPF/DPB Summer Study on High-Energy Physics, Snowmass, Colorado, 1996, edited by D.G. Cassel, L. Trindle Gennari, R.H. Siemann, p. 655; A. Bartl et al., ibid., p. 693; S. Mrenna et al., ibid., p. 681.

[3] M. Carena et al., Rev. Mod. Phys. 71 (1999) 937 arXiv:hep-ex/9712022; hep-ex/9802006.

[4] Atlas Collaboration, Technical Design Report 1999, Vol. II, CERN/LHC/99-15, ATLAS TDR 15. S. Abdullin et al. [CMS Collaboration], J. Phys. G 28 (2002) 469 arXiv:hep-ph/9806366.

[5] E. Accomando et al. [ECFA/DESY LC Physics Working Group Collaboration], Phys. Rept. 299 (1998) 1 arXiv:hep-ph/9705442; H. Murayama and M. E. Peskin, Ann. Rev. Nucl. Part. Sci. 46 (1996) 533 arXiv:hep-ex/9606003; T. Abe et al. [American Linear Collider Working Group Collaboration], in Proc. of the APS/DPF/DPB Summer Study on the Future of Particle Physics (Snowmass 2001) ed. N. Graf, arXiv:hep-ex/0106056 J. A. Aguilar-Saavedra et al. [ECFA/DESY LC Physics Working Group arXiv:hep-ph/0106315, K. Abe et al. [ACFA Linear Collider Working Group Collaboration], arXiv:hep-ph/0109166.

[6] B. Allanach et al., hep-ph/9906224 B. Mukhopadhyaya, S. Roy and F. Vissani, Phys. Lett. B 443, 191 (1998) hep-ph/9808265; F. de Campos et al., Nucl. Phys. B 546, 33 (1999) hep-ph/9710545.

[7] A. Datta, B. Mukhopadhyaya and F. Vissani, Phys. Lett. B 492, 324 (2000) hep-ph/9910296

[8] M. B. Magro et al, JHEP 0309 (2003) 071 hep-ph/0304232.

[9] V. D. Barger, M. S. Berger, P. Ohmann and R. J. N. Phillips, Phys. Rev. D 50 (1994) 4299 hep-ph/9405245.

[10] D. W. Jung, S. K. Kang, J. D. Park and E. J. Chun, JHEP 0408 (2004) 017 hep-ph/0407106.

[11] For a recent review see M. Maltoni, T. Schwetz, M. A. Tortola and J. W. F. Valle, "Status of global fits to neutrino oscillations," New J. Phys. 6, 122 (2004) hep-ph/0405172; Phys. Rev. D 68, 113010 (2003) hep-ph/0309130; Phys. Rev. D 67013011 (2003), hep-ph/0207227.

[12] C. S. Aulakh and R. N. Mohapatra, Phys. Lett. B 119, 136 (1982). L. J. Hall and M. Suzuki, Nucl. Phys. B 231, 419 (1984). G. G. Ross and J. W. Valle, Phys. Lett. B 151, 375 (1985); 
J. R. Ellis et al., Phys. Lett. B 150, 142 (1985).

[13] M. Hirsch et al., Phys. Rev. D 62, 113008 (2000) [Erratum-ibid. D 65, 119901 (2002)] hep-ph/0004115; J. C. Romão et al., Phys. Rev. D 61, 071703 (2000) hep-ph/9907499; M. A. Diaz et al, Phys. Rev. D 68, 013009 (2003) hep-ph/0302021.

[14] S. Y. Choi, E. J. Chun, S. K. Kang and J. S. Lee, Phys. Rev. D 60, 075002 (1999) hep-ph/9903465; D. E. Kaplan and A. E. Nelson, JHEP 0001, 033 (2000) hep-ph/9901254; C. H. Chang and T. F. Feng, Eur. Phys. J. C 12, 137 (2000) hep-ph/9901260; F. Takayama and M. Yamaguchi, Phys. Lett. B 476, 116 (2000) hep-ph/9910320.

[15] M. A. Díaz, J. C. Romão and J. W. Valle, Nucl. Phys. B 524, 23 (1998) hep-ph/9706315

[16] A. Masiero and J. W. Valle, Phys. Lett. B 251, 273 (1990); J. C. Romão, C. A. Santos and J. W. Valle, Phys. Lett. B 288, 311 (1992); J. C. Romão and J. W. Valle, Nucl. Phys. B 381, 87 (1992); K. Huitu, J. Maalampi and K. Puolamaki, Eur. Phys. J. C 6, 159 (1999) hep-ph/9705406; R. Kitano and K. Oda, Phys. Rev. D 61, 113001 (2000) hep-ph/9911327.

[17] M. C. Bento, L. J. Hall and G. G. Ross, Nucl. Phys. B 292, 400 (1987); A. Y. Smirnov and F. Vissani, Nucl. Phys. B 460, 37 (1996) hep-ph/9506416; J. M. Mira et al, Phys. Lett. B 492, 81 (2000) hep-ph/0007266.

[18] F. Takayama and M. Yamaguchi, Phys. Lett. B 485, 388 (2000) hep-ph/0005214.

[19] M. Hirsch and W. Porod, Phys. Rev. D 68, 115007 (2003) arXiv:hep-ph/0307364.

[20] A. Bartl et al., Nucl. Phys. B 600, 39 (2001) hep-ph/0007157, and references therein.

[21] W. Porod et al, Phys. Rev. D 63, 115004 (2001) hep-ph/0011248, and references therein.

[22] M. Hirsch et al, Phys. Rev. D 66 (2002) 095006 hep-ph/0207334.

[23] D. Aristizabal Sierra, M. Hirsch and W. Porod, arXiv:hep-ph/0409241; W. Porod and P. Skands, hep-ph/0401077; A. Bartl, M. Hirsch, T. Kernreiter, W. Porod and J. W. F. Valle, JHEP 0311, 005 (2003) arXiv:hep-ph/0306071; E. J. Chun, D. W. Jung, S. K. Kang and J. D. Park, Phys. Rev. D 66, 073003 (2002) arXiv:hep-ph/0206030; D. Restrepo, W. Porod and J. W. F. Valle, Phys. Rev. D 64, 055011 (2001) arXiv:hep-ph/0104040; M. A. Diaz, D. A. Restrepo and J. W. F. Valle, Nucl. Phys. B 583, 182 (2000) arXiv:hep-ph/9908286.

[24] H. Baer, M. Drees, F. Paige, P. Quintana and X. Tata, Phys. Rev. D 61, 095007 (2000) hep-ph/9906233.

[25] V. D. Barger, C. Kao and T.-J. Li, Phys. Lett. B433, 328 (1998) arXiv:hep-ph/9804451; V. D. Barger and C. Kao, Phys. Rev. D60, 115015 (1999) arXiv:hep-ph/9811489. 
[26] T. Banks, Y. Grossman, E. Nardi and Y. Nir, Phys. Rev. D 52, 531 (1995); J. C. Romão et al., Nucl. Phys. B 482, 3 (1996) hep-ph/9604244; M. Nowakowski and A. Pilaftsis, Nucl. Phys. B 461, 19 (1996) hep-ph/9508271; G. Bhattacharyya, D. Choudhury and K. Sridhar, Phys. Lett. B 355, 193 (1995) hep-ph/9504314; H. P. Nilles and N. Polonsky, Nucl. Phys. B 484, 33 (1997) hep-ph/9606388; B. de Carlos and P. L. White, Phys. Rev. D 55, 4222 (1997) hep-ph/9609443.

[27] W. Porod, Comput. Phys. Commun. 153 (2003) 275 hep-ph/0301101.

[28] P. Skands et al., JHEP 0407 (2004) 036 hep-ph/0311123.

[29] T. Sjostrand, Comput. Phys. Commun. 82 (1994) 74; T. Sjostrand, P. Eden, C. Friberg, L. Lonnblad, G. Miu, S. Mrenna and E. Norrbin, Comput. Phys. Commun. 135 (2001) 238 hep-ph/0010017. 


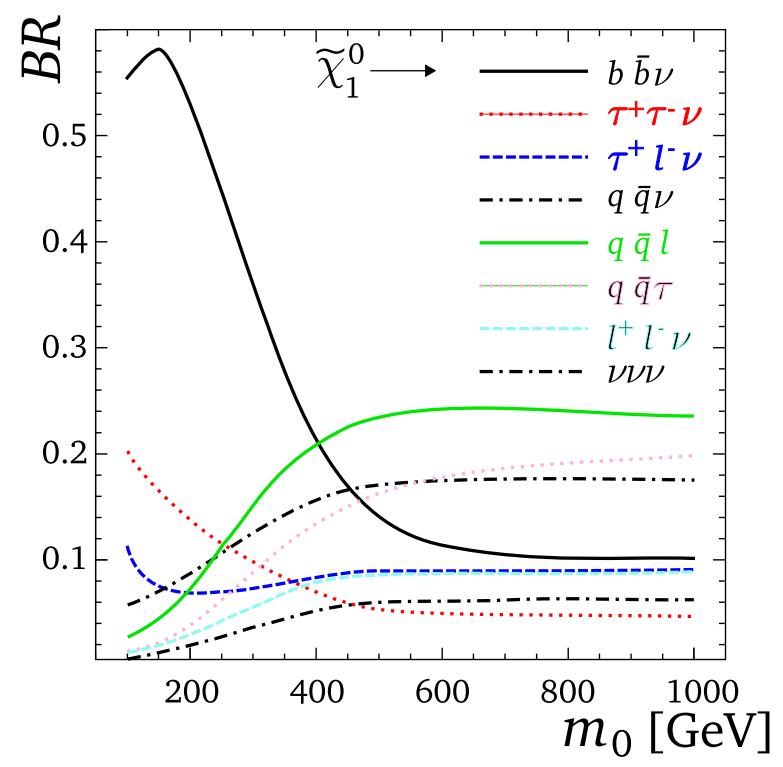

FIG. 1: $\tilde{\chi}_{1}^{0}$ branching ratios versus $m_{0}$ for $A_{0}=-100 \mathrm{GeV}, m_{1 / 2}=350 \mathrm{GeV}, \tan \beta=10$ and $\mu>0$. We took the $\mathrm{R}$-parity violating parameters for each mSUGRA point such that they are compatible with neutrino data and the neutralino decay length is maximized. 


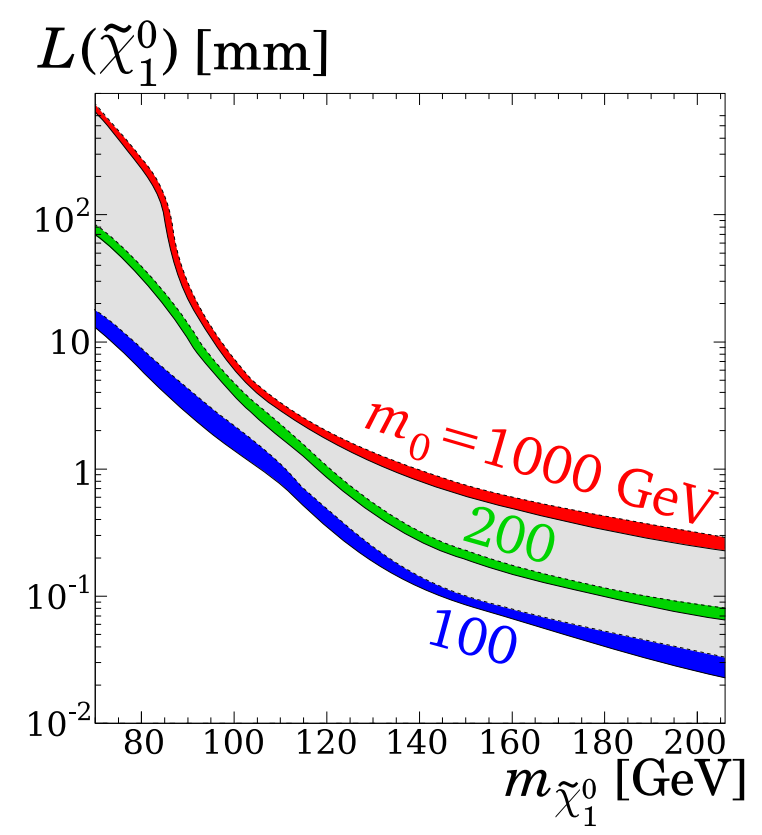

FIG. 2: $\tilde{\chi}_{1}^{0}$ decay length versus $m_{1 / 2}$ for $A_{0}=-100 \mathrm{GeV}, \tan \beta=10$ and $\mu>0$. The width of the $m_{0}$ bands is due to the variation of the $\mathrm{BRpV}$ parameters in such a way that the neutrino masses and mixing angles are within $3 \sigma$ of their best fit values.

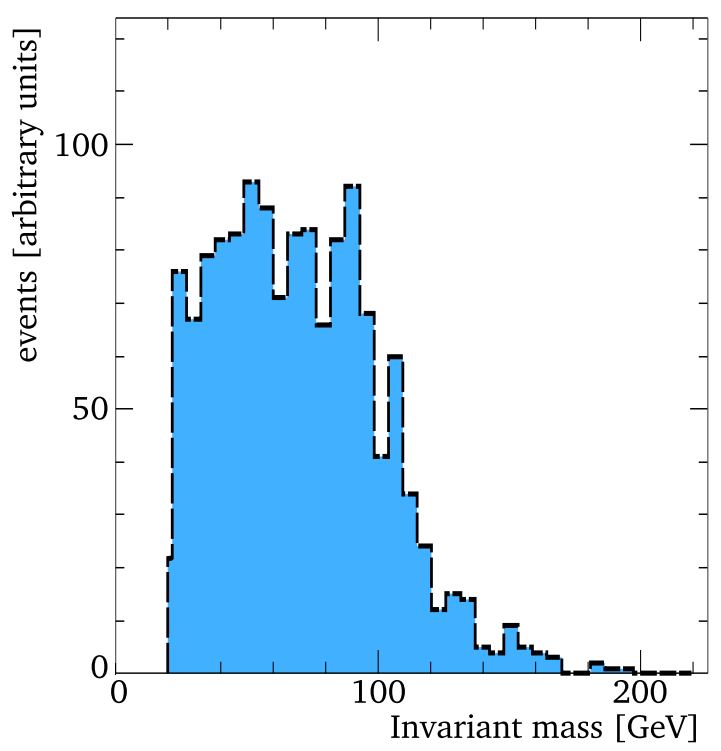

FIG. 3: Reconstructed invariant mass spectrum of displaced vertices after applying the cuts in Sec. III Here we chose $m_{1 / 2}=350 \mathrm{GeV}, m_{0}=400 \mathrm{GeV}, A_{0}=-100 \mathrm{GeV}, \mu>0, \tan \beta=10$, and the $\mathrm{R}$-parity violating parameters for each mSUGRA point such that they are compatible with neutrino data and the neutralino decay length is maximized. 


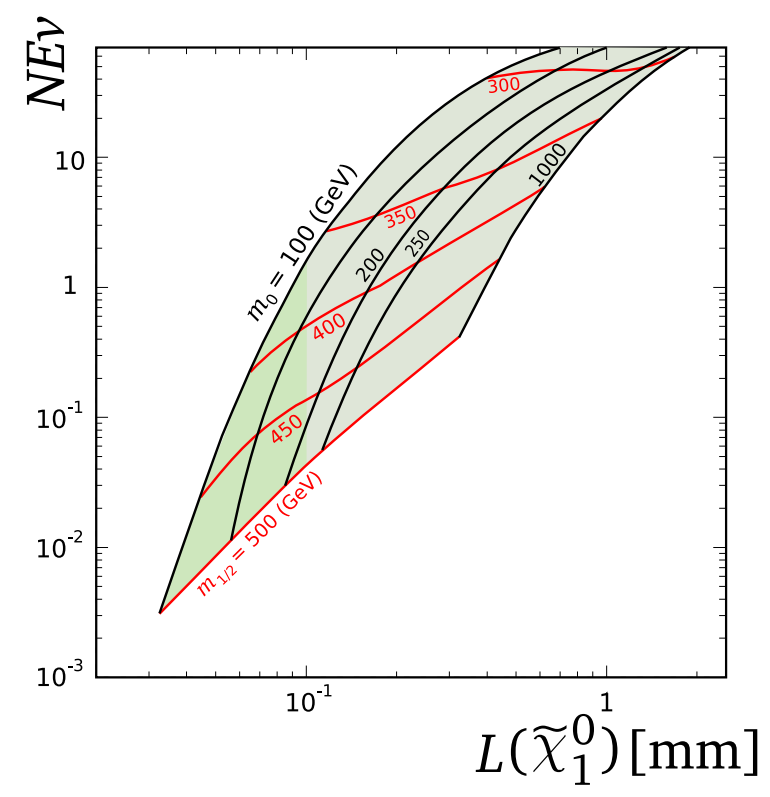

FIG. 4: Expected number of events for an integrated luminosity of $8 \mathrm{fb}^{-1}$ as a function of the neutralino decay length for $A_{0}=-100 \mathrm{GeV}, \mu>0, \tan \beta=10$, and the $\mathrm{R}$-parity violating parameters for each mSUGRA point such that they are compatible with neutrino data and the neutralino decay length is maximized.

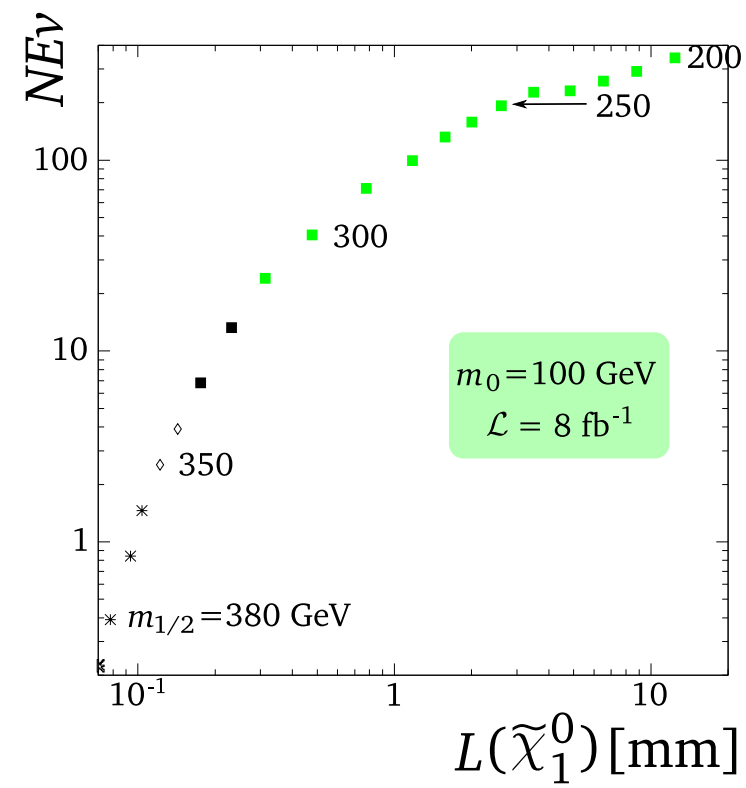

FIG. 5: Same as Fig. 4 but fixing $m_{0}=100 \mathrm{GeV}$. 

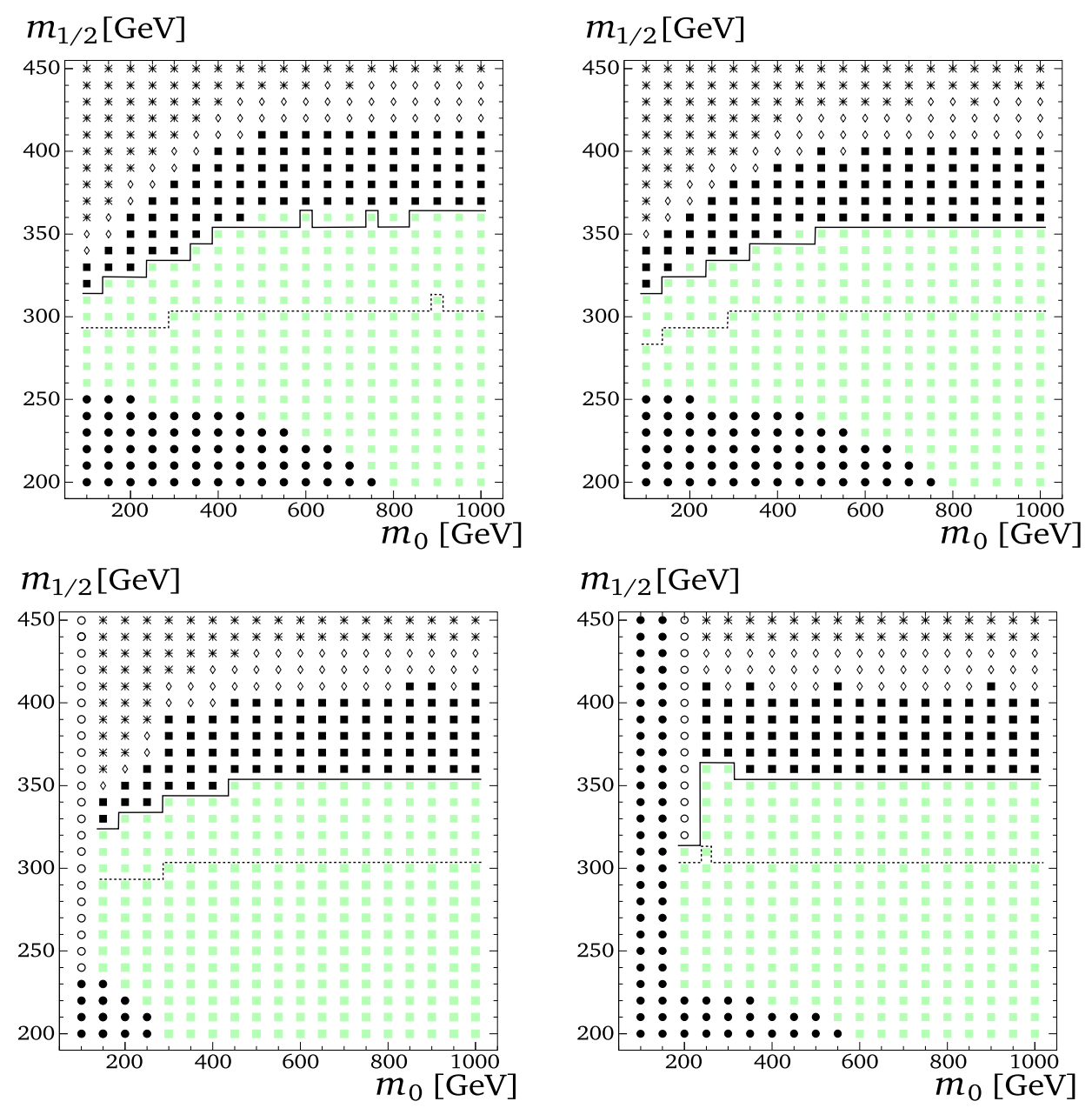

FIG. 6: Reach of Fermilab Tevatron Run II using the displaced vertex signal in the $m_{1 / 2} \otimes m_{0}$ plane for $\mu>0$. In the points below the solid line the expected number of events $(N E v)$ is greater than 4 , while the region below the dashed line exhibits a sizeable statistics $(N E v>15)$. The black squares denote points with an expected number of events greater than 4 for an integrated luminosity of $8 \mathrm{fb}^{-1}$, while the grey (green) squares indicates the points presenting a high statistics $(N E v>15)$ for this luminosity. Moreover, points denoted by diamonds have $2<N E v<4$ with $8 \mathrm{fb}^{-1}$, while the stars correspond to points with $N E v<2$ for $\mathcal{L}=8 \mathrm{fb}^{-1}$. The present direct search limits are violated at the points indicated with a round black circles, while the white circles mark the points where the neutralino is no longer the LSP. The choice of the remaining parameters is the following: In (a) $\tan \beta=10, A_{0}=-100 \mathrm{GeV}$, and the $\mathrm{R}$-parity violating parameters for each mSUGRA point are such that they are compatible with neutrino data and the neutralino decay length is maximized (best case scenario). In (b) we kept the mSUGRA parameters as in (a), however we took the R-parity violating parameters for each mSUGRA point such that they are compatible with neutrino data and the neutralino decay length is minimized (worst case scenario). In (c) the parameters are as in (a) but for $A_{0}=1000 \mathrm{GeV}$. Panel (d) was obtained using the 


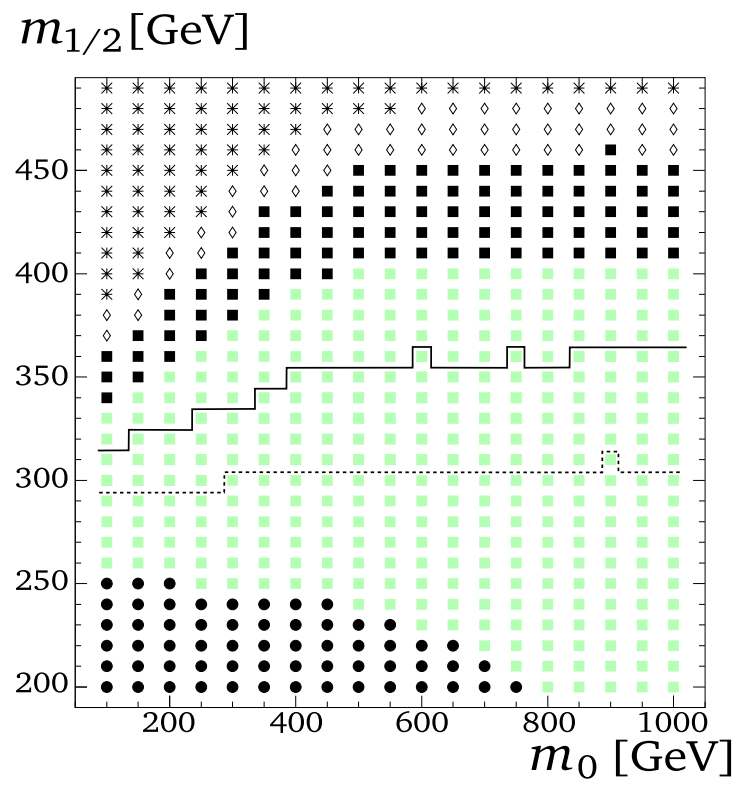

FIG. 7: Reach of the Fermilab Tevatron Run II using the displaced vertex signal in the $m_{1 / 2} \otimes m_{0}$ plane. All the parameters are as in Fig. 6(a) except for an increase in the integrated luminosity from $8 \mathrm{fb}^{-1}$ to $25 \mathrm{fb}^{-1}$.

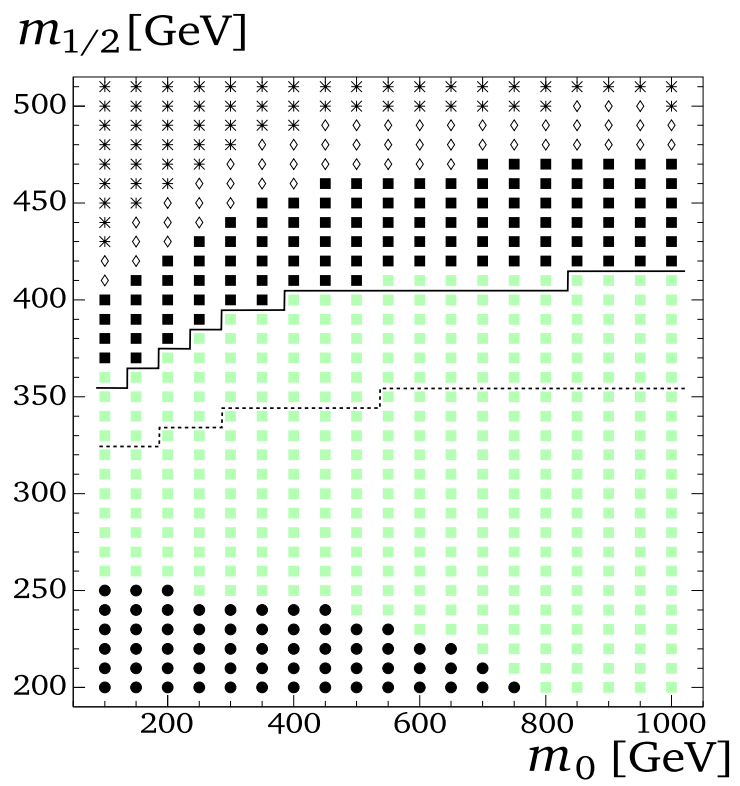

FIG. 8: Reach of Fermilab Tevatron Run II requiring the reconstruction of at least one displaced instead of two displaced vertices. All the parameters are as in Fig. 6(a). 